\title{
Vaccine Development for Zika Virus-Timelines and Strategies
}

\author{
Anna P. Durbin, $\mathrm{MD}^{1}$ \\ ${ }^{1}$ Department of International Health, Center for Immunization \\ Research, Johns Hopkins Bloomberg School of Public Health, \\ Baltimore, Maryland
}

Semin Reprod Med 2016;34:299-304

\begin{abstract}
Address for correspondence Anna P. Durbin, MD, Department of International Health, Center for Immunization Research, Johns Hopkins Bloomberg School of Public Health, Baltimore, MD 21205 (e-mail: adurbin1@jhu.edu).
\end{abstract}

\begin{abstract}
Keywords

- Zika virus

- vaccines

- DNA vaccine

Zika virus is a mosquito-borne Flavivirus that spread rapidly through South and Central America in 2015 to 2016. Microcephaly has been causally associated with Zika virus infection during pregnancy and the World Health Organization declared Zika virus as a Public Health Emergency of International Concern. To address this crisis, many groups have expressed their commitment to developing a Zika virus vaccine. Different strategies for Zika virus vaccine development are being considered including recombinant live attenuated vaccines, purified inactivated vaccines (PIVs), DNA vaccines, and viral vectored vaccines. Important to Zika virus vaccine development will be the target group chosen for vaccination and which end point(s) is chosen for efficacy determination. The first clinical trials of Zika virus vaccine candidates will begin in Q3/4 2016 but the pathway to licensure for a Zika virus vaccine is expected to take several years. Efforts are ongoing to accelerate Zika virus vaccine development and evaluation with the ultimate goal of reducing time to licensure.
\end{abstract}

Zika virus was first isolated from the blood of a sentinel rhesus macaque in the Zika forest of Uganda in $1947 .{ }^{1}$ Sporadic reports of Zika virus infection and/or serologic evidence of Zika virus infection were reported from 1951 to 1981 from Africa and Asia. ${ }^{2}$ The first major outbreak of Zika virus was reported from the Island of Yap in Micronesia in 2007 where it was estimated that $72.6 \%$ of the population $\geq 3$ years of age was infected. ${ }^{3}$ The majority of symptomatic patients presented with rash (90\%), arthritis/arthralgia (65\%), and fever (65\%). Only approximately $19 \%$ of subjects found to be seropositive to Zika virus in a serosurvey recounted being symptomatic with a Zika-like illness. No cases of microcephaly or Guillain-Barré syndrome (GBS) were reported from that outbreak but the population of the island was small ( $<7,000$ persons, $\geq 3$ years of age). In October 2013, the largest outbreak of Zika virus recorded up to that time began in French Polynesia. ${ }^{4,5}$ It was estimated that 28,000 Zika virus infections occurred ( $\sim 11 \%$ of the population) with most infections presenting low-grade fever, rash, arthralgia, and conjunctivitis. Evidence of perinatal Zika virus infection and
Zika virus infection complicated by GBS were documented. ${ }^{6,7}$ In retrospective analyses of the French Polynesian outbreak, an association between GBS and Zika virus infection as well as Zika virus infection during the first and/or second trimester and microcephaly were reported. ${ }^{8,9}$

Brazil first identified an outbreak of Zika virus in its northeast region in early 2015. By September, increasing numbers of cases of microcephaly were being reported, particularly from regions involved in the Zika virus outbreak. ${ }^{10}$ Evidence of a link between Zika virus infection in pregnancy, particularly during the first trimester, and microcephaly in the fetus mounted ${ }^{11-13}$ and a causal association between Zika virus infection and microcephaly and/or other birth defects was formally established. ${ }^{14}$ The majority of women in whom microcephaly of the fetus was diagnosed reported some symptoms of Zika virus infection at some point during their pregnancy, usually in the first or second trimester; rash was the most common symptom reported. A recent report from Colombia reported four fetuses with microcephaly who were born to women with laboratory evidence of congenial Zika
Issue Theme The Zika Virus Pandemic and Reproductive Medicine; Guest Editors, Jeanne Sheffield, MD and James Segars, MD
Copyright $\odot 2016$ by Thieme Medical Publishers, Inc., 333 Seventh Avenue, New York, NY 10001, USA. Tel: +1(212) 584-4662.
DOI http://dx.doi.org/ 10.1055/s-0036-1592070. ISSN 1526-8004. 
virus infection but who did not report symptoms of Zika virus during their pregnancy. ${ }^{15}$

A link between GBS and Zika virus infection is less well established. The strongest evidence of such a link is from a retrospective case-control study of GBS cases identified during a Zika virus outbreak in French Polynesia in $2013 .{ }^{9}$ Forty-two cases of GBS were identified with $88 \%$ of those reporting a history of recent viral syndrome, a median of 6 days before the onset of neurological symptoms. Serum from the GBS patients was tested by reverse transcription polymerase chain reaction (RT-PCR) for Zika virus during the hospital admission but was negative in all cases. Although serum from these patients was eventually found to be positive for Zika virus antibody by either immunoglobulin M, immunoglobulin $\mathrm{G}(\operatorname{IgG})$, or neutralization test, samples from these patients was taken up to 3 months posthospitalization, and it is unclear at what time the sample became positive for Zika virus. In contrast, serum from 98 matched control patients hospitalized with a nonfebrile illness was collected at one time point within 7 days of the admission date of the matched GBS case. All 42 GBS patients were found to have neutralizing antibody against Zika virus compared with 54/98 (56\%) of control patients. Although sera from ix patients demonstrated high reactivity toward asialo-GM1 ganglioside (GA1), further testing did not demonstrate any competition between GA1 and Zika virus proteins suggesting that there was no molecular mimicry between Zika virus antigens and GA1.

Aside from the congenital birth defects caused by Zika virus infection during pregnancy, the virus causes only a mild infection characterized by rash, low-grade fever, nonpurulent conjunctivitis, and myalgia with the majority of those infected not reporting symptoms. ${ }^{3,12}$ Complicating Zika virus control programs, there have been several reports of sexual transmission of Zika virus. ${ }^{16-19}$ In the cases reported to date, all men who transmitted Zika virus to their sexual partners were symptomatic. Zika virus has been detected in semen by RT-PCR up to 62 days after symptoms have developed. ${ }^{20}$ It is not currently known how long infectious virus can remain in semen and be transmitted to sexual partners. The current recommendation is that men who had a symptomatic Zika virus infection refrain from sexual activity or use a condom for 6 months after illness to prevent transmission of Zika virus to their sexual partner. ${ }^{18}$

\section{Considerations for Vaccine Development}

In response to the link between Zika virus infection during pregnancy and microcephaly, the World Health Organization (WHO) declared Zika virus a Public Health Emergency of International Concern, February 1, 2016. Vector control, personal protection against mosquitoes, and pregnancy prevention are currently the only available approaches to reduce the risk of fetal infection in Zika-endemic areas. A majority priority of the global public health community is the development of a safe and effective Zika virus vaccine to address the dire need for additional prevention and control methods. Several factors related to Zika virus infection may complicate the development and evaluation of candidate vaccines. These include the low incidence of Zika illness, short period of viremia, the risk of microcephaly caused by congenital Zika virus infection, the risk of sexual transmission, and the potential link of Zika virus and GBS.

A list of companies, research organizations, and academic institutions that have expressed interest in developing a Zika virus vaccine is found in - Table 1. This list is based on informal discussions and presentations at conferences. The majority of the candidates that are presented in - Table $\mathbf{1}$ are based on vaccines that have been developed for other related flaviviruses such as dengue virus (DENV), yellow fever virus (YFV), West Nile virus, Japanese encephalitis virus (JEV), and tick-borne encephalitis virus. Important considerations for Zika virus vaccine development are (1) the target population for vaccination, (2) the number of doses required to induce an immune response, (3) the effect of previous Flavivirus exposure on Zika virus vaccine safety and immunogenicity, (4) previous human experience with related types of vaccines/ adjuvants, and (5) sustainability.

During an active outbreak of Zika virus, the population targeted for vaccination may be different from the target population in a nonoutbreak setting. Ultimately, it is hoped that a licensed Zika virus vaccine will be incorporated into current vaccination programs of infants and children. The model of this is the rubella vaccine which has led to the near eradication of congenital rubella syndrome throughout the Americas. It is unlikely that there will be an adequate supply of a licensed Zika virus vaccine to vaccinate all children in endemic areas for several years after licensure. In an outbreak setting and until adequate supplies of vaccine are available, it is probable that an available vaccine will be prioritized to those at highest risk-women of child-bearing age and perhaps pregnant women in Zika-endemic areas. Only a limited number of vaccines would be considered for evaluation in pregnant women (killed or subunit protein vaccine) as other types of vaccines may pose a safety risk (live vaccines) or there are no data available regarding the use of this type of vaccine in this population (DNA vaccine, RNA vaccine, vectored vaccines). Vaccination of pregnant women as a way to prevent congenital microcephaly would be difficult as the highest risk occurs with Zika virus infection during the first trimester. ${ }^{21}$ Vaccination would have to occur very early in pregnancy and the vaccine would have to produce a protective response quickly and following a minimum of doses (preferably one). The WHO is in the process of developing separate target product profiles for vaccines to be used either in an outbreak setting or as part of a routine immunization program. ${ }^{22}$ These profiles will help aid vaccine manufacturers as they move forward with development of candidate vaccines.

Zika virus is a Flavivirus and is related to other flaviviruses such as DENV, YFV, and JEV. The phenomenon of antibodydependent enhancement of infection in which antibodies to one DENV serotype cross-react with a second different DENV serotype and allow the virus to enter monocytes and macrophages through the $\mathrm{Fcr}$ receptor. ${ }^{23,24}$ Epidemiological studies of dengue have demonstrated a higher incidence of severe dengue with secondary dengue infection and with higher 
Table 1 Proposed Zika virus vaccine candidates/platforms

\begin{tabular}{|l|l|l|}
\hline Type & Candidate & Status \\
\hline \multirow{5}{*}{ Inactivated } & PaxVax, California & Preclinical \\
\cline { 2 - 3 } & NewLink Genetics, Massachusetts & Preclinical \\
\cline { 2 - 3 } & GSK, United States/Belgium & Preclinical \\
\cline { 2 - 3 } & Bharat Biotech, India & Preclinical \\
\cline { 2 - 3 } & WRAIR/Sanofi Pasteur, United States and France & Phase 1: 2016-2017 \\
\hline \multirow{5}{*}{ Subunit/peptide } & Protein Sciences, Connecticut & Preclinical \\
\cline { 2 - 3 } & Hawaii Biotech, Hawaii & Preclinical \\
\cline { 2 - 3 } & Bharat Biotech, India & Preclinical \\
\cline { 2 - 3 } & Replikins, Massachusetts & Phase 1: Q4 2016 \\
\hline \multirow{5}{*}{ Live } & NIAID-LID/Instituto Butantan, United States/Brazil & Preclinical \\
\cline { 2 - 3 } & UTMB/Instituto Evandro Chagas, United States/Brazil & Preclinical \\
\cline { 2 - 3 } & Sanofi Pasteur, France & Preclinical \\
\hline \multirow{5}{*}{ Vectored } & Jenner Institute (chimpanzee adenovirus), UK & Preclinical \\
\cline { 2 - 3 } & Harvard University (VSV), Massachusetts & Preclinical \\
\cline { 2 - 3 } & Themis Bioscience (measles), Austria & Phase 1: Q3 2016 \\
\hline \multirow{5}{*}{ DNA/RNA } & NIAID-VRC (Biojector needle-free), United States & Phase 1: Q3 2016 \\
\cline { 2 - 3 } & Inovio Pharmaceuticals (electroporation), Pennsylvania & Preclinical \\
\cline { 2 - 3 } & GSK (RNA), United States/Belgium & \\
\hline
\end{tabular}

Abbreviation: VRC, Vaccine Research Center; VSV, vesicular stomatitis virus.

virus titer. ${ }^{25-28}$ In vitro enhancement of Zika virus by DENV antibodies was observed in early studies of Zika virus ${ }^{29}$ and has been repeated recently. ${ }^{30}$ The cross-reactivity between Zika virus and DENV antibodies has also made the diagnosis of Zika virus more difficult in dengue-endemic areas. Despite this cross-reactivity, Zika virus infection does not appear to be more severe in dengue-endemic areas nor do levels of viremia appear to be higher. However, it is unclear at this time whether or not cross-reactive antibodies are contributing to the transmission of Zika virus or somehow affecting infection of the fetus.

Whether or not, preexisting DENV antibodies will affect the safety and/or immunogenicity of a Zika virus vaccine is unknown. Seroconversion rates to the live attenuated DENV vaccine CYD-TDV (Dengvaxia, Sanofi Pasteur, Lyon, France) were higher in dengue exposed and YFV vaccinated individuals. ${ }^{31-33}$ Efficacy of the vaccine was also significantly higher in persons who were dengue exposed at the time of first vaccination compared with those who were dengue naïve. ${ }^{34}$ Despite improved immunogenicity in those with preexisting dengue antibody, there was no increase in the reactogenicity of the vaccine during the immediate postvaccination period. Studies of candidate Zika virus vaccines will have to be done in both Flavivirus-naïve and Flavivirus-exposed individuals to better assess the effect of preexisting antibody on vaccine safety and immunogenicity. An important objective of any Phase 3 study of candidate Zika virus vaccines should be to determine if there are differences in the short- and long-term safety and efficacy of candidate Zika virus vaccines depending on Flavivirus serostatus at the time of vaccination. In addition, should a link between Zika virus infection and GBS be determined, the incidence of GBS during clinical trials and postvaccine licensure must be carefully monitored.

\section{Vaccine Platforms}

The platforms being employed by Zika virus vaccine developers are quite varied (see - Table 1). ${ }^{35}$ Most are based on other Flavivirus candidate vaccines and include live attenuated virus vaccines, whole inactivated virus vaccines, and subunit protein vaccines. Newer strategies such as DNA vaccines and as viral-vectored vaccines are also under development. Two Zika virus vaccines have recently demonstrated protection in preclinical mouse studies. A DNA vaccine encoding the full-length prM and E proteins (prM-E) of the Brazil BeH815744 Zika virus strain was developed by researchers at the Harvard Medical School, the Massachusetts Institute of Technology, the University of Sao Paulo, and the Walter Reed Institute of Research. This vaccine was evaluated in Balb/c mice. ${ }^{36}$ Mice inoculated intramuscularly (IM) with $50 \mu \mathrm{g}$ of the DNA vaccine elicited higher anti-E antibody titers than did the same vaccine administered subcutaneously (SQ) or than DNA vaccines that did not encode the prM protein. A single dose of the prM-E DNA vaccine induced complete protection in Balb/c mice against challenge with $10^{5}$ virus particles of either one of two wild-type Zika viruses of Asian lineage given at 4 or 8 weeks postvaccination. DNA vaccine constructs that did not include prM or full-length E did not elicit complete protection against challenge. IgG purified from vaccinated Balb/c and then infused intravenously was 
also able to protect recipient mice from challenge when the titer of anti-E antibody was $\geq 2.35 \log _{10}$. Mice vaccine with the prM-E DNA vaccine and then depleted of $\mathrm{CD} 4^{+}$and $\mathrm{CD} 8^{+} \mathrm{T}$ cells on day 2 and day 1 prior to challenge were also protected. These studies demonstrated that protection against wildtype Zika virus challenge can be mediated by antibody alone and that protection was correlated with the magnitude of the antibody response. ${ }^{36}$ These same investigators also evaluated the immunogenicity and protective efficacy of PIV derived from the Puerto Rico PRVAB59 strain of Zika virus. A $1 \mu \mathrm{g}$ dose of PIV adjuvanted with alum was delivered either IM or SQ to Balb/c mice (five mice per group). Higher antibody titers were elicited when the vaccine was given by the IM route and all mice in this group were completely protected against challenge with wild-type Zika virus. Two of five mice that received the vaccine SQ had detectable levels of viremia following challenge, although viremia was of lower titer than in the control group. These results are encouraging and warrant further evaluation of these candidates in humans and to this end, Sanofi Pasteur is partnering with WRAIR for further development and evaluation of the PIV Zika virus vaccine.

The U.S. National Institutes of Health (NIH) is developing two Zika virus vaccines. The Laboratory of Infectious Diseases (LID) of the NIH is developing a live-attenuated chimeric Zika virus vaccine based on one of the components of its live attenuated tetravalent dengue vaccine currently in Phase 3 clinical trial in Brazil. ${ }^{37,38}$ The candidate Zika virus vaccine virus is comprised the prM and E proteins of Zika virus and the nonstructural proteins of the DENV-2. It is expected to begin Phase 1 clinical evaluation in Flavivirus-naïve subjects in the fourth quarter of 2016. Should the vaccine prove to be immunogenic in Phase 1 clinical evaluation, the LID hopes to achieve an early efficacy evaluation of the vaccine using a Zika virus human challenge model that is currently under development. Ultimately, the LID hopes to combine this Zika virus vaccine with their live attenuated tetravalent virus (LATV) dengue vaccine to create a pentavalent vaccine that would be administered to children in endemic areas. They are partnering with the Instituto Butantan which has licensed the LATV dengue vaccine and is currently conducting the Phase 3 trial of the dengue vaccine. The Vaccine Research Center of the NIH is developing a DNA Zika virus vaccine based on the technology it used for a highly immunogenic West Nile virus vaccine. ${ }^{39}$ This vaccine is expected to begin human clinical trials in late Q3 or early Q4 2016.

Inovio Pharmaceuticals (Pennsylvania), in collaboration with the University of Pennsylvania, the Wistar Institute of Philadelphia, and GeneOne Life Science of South Korea is developing a DNA vaccine for Zika virus, GLS-5700. ${ }^{40}$ The vaccine elicited an immune response against Zika virus in rabbits and monkeys and the manufacturer has submitted an investigational new drug application to the U.S. FDA. It will begin a Phase 1 clinical trial in approximately 40 volunteers in the United States and Canada in Q3 2016 with safety and immunogenicity results expected by the end of the year. The vaccine is given intradermally followed by electroporation and will evaluate the safety, tolerability, and immunogenicity of the vaccine in dengue-naïve subjects. GLS-5700 is the first
Zika virus vaccine to receive approval from the FDA to begin Phase 1 clinical evaluation and is registered on Clinicaltrials. gov (NCT02809443).

Should these and other candidate vaccines demonstrate an acceptable safety and immunogenicity profile in denguenaïve subjects, they would then be further tested in endemic areas to evaluate the effect of preexisting dengue (or other Flavivirus) immunity on the safety and immunogenicity of the candidate vaccine. More novel vaccine platforms such as DNA vaccines would need to be more carefully evaluated for use in pregnant women if the licensure of the vaccine were to include this population. A DNA vaccine has yet to be licensed for use in people, pregnant or otherwise and as such, would have to overcome this regulatory hurdle.

\section{Clinical Trial End Points}

Several Zika virus vaccine candidates are expected to begin Phase 1 clinical evaluation in 2016. Should any of these candidates look promising, they would then undergo expanded safety and immunogenicity evaluation in Zika-endemic areas (Phase 2 clinical trial) followed by a formal efficacy trial (Phase 3 ) in preparation for licensure. A critical question in this process will be what end point chosen for efficacy analysis; prevent of infection, prevention of disease, prevention of microcephaly, or a nonclinical end point such as an immune correlate.

It is unlikely that the prevention of microcephaly will be a suitable end point as it would require a very large sample size (assuming vaccination of women of child-bearing age) and the primary end point may not be reached for years. The primary efficacy outcome in this scenario would be dependent on the birth rate within the study population which may be affected during an active Zika virus outbreak. Prevention of clinical infection or clinical disease would be a preferable primary efficacy outcome; however, both of these may be problematic to achieve. With only approximately $18 \%$ of infected individuals developing symptomatic illness, ${ }^{3}$ prevention of disease as an efficacy end point would require a very large sample size. In addition, of those cases who have been symptomatic, fever (objective or subjective) was reported in 28 to $73 \%$ of cases, ${ }^{3,12,41}$ making illness more difficult to recognize using this criteria. In contrast, rash was reported in 77 to $100 \%$ of cases. For this reason, enhanced surveillance with rash as the impetus for testing for confirmation of Zika virus infection may be more successful as a trigger for case investigation. Although Zika virus is detected in the blood for only a few days following the onset of rash, Zika viral antigen can be detected in the urine by PCR for up to 2 weeks post-onset of rash. ${ }^{42}$ For this reason, using infection with Zika virus as the primary end point for efficacy in a clinical trial, as defined by the detection of Zika virus antigen in urine or saliva, may be possible. This would require relatively frequent ( $\sim$ every 2 weeks) sample collection from enrolled subjects, but this end point would require a much smaller sample than prevention of clinical disease as an end point, and an efficacy determination may be reached much earlier. 
Zika virus has spread through South and Central America at an alarming rate. However, it is not clear if Zika virus transmission will be sustained or if, as the population becomes less susceptible due to the current outbreak, Zika virus transmission will rapidly decline as was seen with West Nile virus infection in North America. Should this occur, there may not be ongoing transmission of Zika virus by the time a vaccine candidate is ready to begin a Phase 3 efficacy evaluation. This was evident in the most recent Ebola outbreak in West Africa and may make it very difficult to determine if a candidate Zika virus vaccine is efficacious. Should this occur or should clinical infection/disease not be feasible as an end point, the ability of the vaccine to induce neutralizing antibody in Flavivirus-naïve individuals could potentially be used. Neutralizing antibody titers in Flavivirus-naïve subjects would be evaluated first as the antibody titers induced by the vaccine in those already exposed to other flaviviruses such as dengue or yellow fever may be higher due to crossreactivity and may not be indicative of protection. ${ }^{43} \mathrm{~A}$ Zika virus human challenge model could help establish an immune correlate of protection and could potentially determine the titer of antibody required for protection.

\section{Conclusion}

In summary, more than 15 groups have committed to the development of a Zika virus vaccine with the first clinical trials expected to begin in Q3 2016. Even with efforts to condense the timeline to introduction of a vaccine, the pathway to licensure will likely take a minimum of 4 to 5 years to complete. Frequent consultation with regulatory authorities in affected regions, development of better diagnostics, and enhanced surveillance for Zika virus in preparation of vaccine efficacy trials will be important to minimize delays in the licensing and deployment of a Zika virus vaccine. Postlicensure, surveillance for GBS and other unexpected adverse events such as enhanced disease, will need to be in place with the ability to link the occurrence of such events to vaccination status. A safe and effective vaccine would be a valuable tool for the prevention and control of Zika virus infection. Its development will require adequate funding and a commitment beyond the current outbreak. The ultimate goal of these efforts will be the inclusion of a licensed vaccine into the routine childhood immunization program of all Zikaaffected regions.

\section{References}

1 Dick GW, Kitchen SF, Haddow AJ. Zika virus. I. Isolations and serological specificity. Trans R Soc Trop Med Hyg 1952;46(5): 509-520

2 Hayes EB. Zika virus outside Africa. Emerg Infect Dis 2009;15(9): 1347-1350

3 Duffy MR, Chen TH, Hancock WT, et al. Zika virus outbreak on Yap Island, Federated States of Micronesia. N Engl J Med 2009;360(24): 2536-2543

4 Cao-Lormeau VM, Roche C, Teissier A, et al. Zika virus, French Polynesia, South Pacific, 2013. Emerg Infect Dis 2014;20(6): 1085-1086
5 Musso D, Nilles EJ, Cao-Lormeau VM. Rapid spread of emerging Zika virus in the Pacific area. Clin Microbiol Infect 2014;20(10): O595-0596

6 Besnard M, Lastere S, Teissier A, Cao-Lormeau V, Musso D. Evidence of perinatal transmission of Zika virus, French Polynesia, December 2013 and February 2014. Euro Surveill 2014;19(13):

7 Oehler E, Watrin L, Larre P, et al. Zika virus infection complicated by Guillain-Barre syndrome-case report, French Polynesia, December 2013. Euro Surveill 2014;19(9):

8 Cauchemez S, Besnard M, Bompard P, et al. Association between Zika virus and microcephaly in French Polynesia, 2013-15: a retrospective study. Lancet 2016;387(10033):2125-2132

9 Cao-Lormeau V-M, Blake A, Mons S, et al. Guillain-Barré syndrome outbreak associated with Zika virus infection in French Polynesia: a case-control study. Lancet 2016;387(10027):1531-1539

10 Schuler-Faccini L, Ribeiro EM, Feitosa IM, et al; Brazilian Medical Genetics Society-Zika Embryopathy Task Force. Possible association between Zika virus infection and microcephaly - Brazil, 2015. MMWR Morb Mortal Wkly Rep 2016;65(3):59-62

11 Mlakar J, Korva M, Tul N, et al. Zika virus associated with microcephaly. N Engl J Med 2016;374(10):951-958

12 Brasil P, Pereira JP Jr, Raja Gabaglia C, et al. Zika virus infection in pregnant women in Rio de Janeiro - preliminary report. N Engl J Med 2016. doi 10.1056/NEJMoa1602412

13 Kleber de Oliveira W, Cortez-Escalante J, De Oliveira WT, et al. Increase in reported prevalence of microcephaly in infants born to women living in areas with confirmed Zika virus transmission during the first trimester of pregnancy - Brazil, 2015. MMWR Morb Mortal Wkly Rep 2016;65(9):242-247

14 Rasmussen SA, Jamieson DJ, Honein MA, Petersen LR. Zika virus and birth defects-reviewing the evidence for causality. N Engl J Med 2016;374(20):1981-1987

15 Pacheco O, Beltrán M, Nelson CA, et al. Zika virus disease in Colombia - preliminary report. N Engl J Med 2016. doi 10.1056/ NEJMoa 1604037

16 Oster AM, Brooks JT, Stryker JE, et al. Interim guidelines for prevention of sexual transmission of Zika virus - United States, 2016. MMWR Morb Mortal Wkly Rep 2016;65(5):120-121

17 Hills SL, Russell K, Hennessey M, et al. Transmission of Zika virus through sexual contact with travelers to areas of ongoing transmission - continental United States, 2016. MMWR Morb Mortal Wkly Rep 2016;65(8):215-216

18 Oster AM, Russell K, Stryker JE, et al. Update: interim guidance for prevention of sexual transmission of Zika virus - United States, 2016. MMWR Morb Mortal Wkly Rep 2016;65(12):323-325

19 Foy BD, Kobylinski KC, Chilson Foy JL, et al. Probable non-vectorborne transmission of Zika virus, Colorado, USA. Emerg Infect Dis 2011;17(5):880-882

20 Atkinson B, Hearn P, Afrough B, et al. Detection of Zika virus in semen. Emerg Infect Dis 2016;22(5):940

21 Johansson MA, Mier-y-Teran-Romero L, Reefhuis J, Gilboa SM, Hills SL. Zika and the risk of microcephaly. N Engl J Med 2016;375(1): $1-4$

22 WHO. WHO and experts prioritize vaccines, diagnostics, and innovative vector control tools for Zika R\&D. 2016. Available at: http://www.who.int/mediacentre/news/notes/2016/research-development-zika/en/. Accessed June 30, 2016

23 Halstead SB, O'Rourke EJ. Antibody-enhanced dengue virus infection in primate leukocytes. Nature 1977;265(5596):739-741

24 Kliks SC, Nisalak A, Brandt WE, Wahl L, Burke DS. Antibodydependent enhancement of dengue virus growth in human monocytes as a risk factor for dengue hemorrhagic fever. Am J Trop Med Hyg 1989;40(4):444-451

25 Guzmán MG, Kourí G, Valdés L, Bravo J, Vázquez S, Halstead SB. Enhanced severity of secondary dengue-2 infections: death rates in 1981 and 1997 Cuban outbreaks. Rev Panam Salud Publica 2002;11(4):223-227 
26 Kliks SC, Nimmanitya S, Nisalak A, Burke DS. Evidence that maternal dengue antibodies are important in the development of dengue hemorrhagic fever in infants. Am J Trop Med Hyg 1988; 38(2):411-419

27 Burke DS, Nisalak A, Johnson DE, Scott RM. A prospective study of dengue infections in Bangkok. Am J Trop Med Hyg 1988;38(1): 172-180

28 Vaughn DW, Green S, Kalayanarooj S, et al. Dengue viremia titer, antibody response pattern, and virus serotype correlate with disease severity. J Infect Dis 2000;181(1):2-9

29 Fagbami AH, Halstead SB, Marchette NJ, Larsen K. Cross-infection enhancement among African flaviviruses by immune mouse ascitic fluids. Cytobios 1987;49(196):49-55

30 Dejnirattisai W, Supasa P, Wongwiwat W, et al. Dengue virus serocross-reactivity drives antibody-dependent enhancement of infection with Zika virus. Nat Immunol 2016. doi 10.1038/ni.3515

31 Guirakhoo F, Kitchener S, Morrison D, et al. Live attenuated chimeric yellow fever dengue type 2 (ChimeriVax-DEN2) vaccine: phase I clinical trial for safety and immunogenicity: effect of yellow fever pre-immunity in induction of cross neutralizing antibody responses to all 4 dengue serotypes. Hum Vaccin 2006; 2(2):60-67

32 Poo J, Galan F, Forrat R, Zambrano B, Lang J, Dayan G. Liveattenuated tetravalent dengue vaccine in dengue-naïve children, adolescents, and adults in Mexico City: randomized controlled phase 1 trial of safety and immunogenicity. Pediatr Infect Dis J 2011;30(1):e9-e17

33 Capeding RZ, Luna IA, Bomasang E, et al. Live-attenuated, tetravalent dengue vaccine in children, adolescents and adults in a dengue endemic country: randomized controlled phase I trial in the Philippines. Vaccine 2011;29(22): 3863-3872
34 Hadinegoro SR, Arredondo-García JL, Capeding MR, et al; CYD-TDV Dengue Vaccine Working Group. Efficacy and long-term safety of a dengue vaccine in regions of endemic disease. N Engl J Med 2015; 373(13):1195-1206

35 WHO. WHO global consultation of research related to Zika virus infection. Paper Presented at: WHO Global Consultation of Research Related to Zika Virus Infection; Geneva; March 7-9, 2016

36 Larocca RA, Abbink P, Peron JP, et al. Vaccine protection against Zika virus from Brazil. Nature 2016. doi 10.1038/nature18952

37 Kirkpatrick BD, Durbin AP, Pierce KK, et al. Robust and balanced immune responses to all 4 dengue virus serotypes following administration of a single dose of a live attenuated tetravalent dengue vaccine to healthy, Flavivirus-naive adults. J Infect Dis 2015;212(5):702-710

38 Kirkpatrick BD, Whitehead SS, Pierce KK, et al. The live attenuated dengue vaccine TV003 elicits complete protection against dengue in a human challenge model. Sci Transl Med 2016;8(330):330ra36

39 Ledgerwood JE, Pierson TC, Hubka SA, et al; VRC 303 Study Team. A West Nile virus DNA vaccine utilizing a modified promoter induces neutralizing antibody in younger and older healthy adults in a phase I clinical trial. J Infect Dis 2011;203(10):1396-1404

40 Dyer O. Trials of Zika vaccine are set to begin in North America. BMJ 2016;353:i3588

41 Thomas DL, Sharp TM, Torres J, et al. Local transmission of Zika virus-Puerto Rico, November 23, 2015-January 28, 2016. MMWR Morb Mortal Wkly Rep 2016;65(6):154-158

42 Gourinat AC, O'Connor O, Calvez E, Goarant C, Dupont-Rouzeyrol M. Detection of Zika virus in urine. Emerg Infect Dis 2015;21(1): 84-86

43 Lanciotti RS, Kosoy OL, Laven JJ, et al. Genetic and serologic properties of Zika virus associated with an epidemic, Yap State, Micronesia, 2007. Emerg Infect Dis 2008;14(8):1232-1239 